\title{
CDISC Interventions Class
}

National Cancer Institute

\section{Source}

National Cancer Institute. CDISC Interventions Class. NCI Thesaurus. Code C103374.

This SDT M class captures investig ational, therapeutic and other treatments that are administered to the subject (with some actual or expected physiological effect) either as specified by the study protocol (e.g., exposure to study drug), coincident with the study assessment period (e.g., concomitant medications), or self-administered by the subject (such as use of alcohol, tobacco, or caffeine). 